\title{
Monitoring the degradation of lubricating oil by means of surface plasmon
}

\author{
J.A. Heredia-Cancino ${ }^{1}$, F. Félix-Domínguez ${ }^{2}$, R. C. Carrillo-Torres ${ }^{3}$ and M. E. Álvarez-Ramos ${ }^{3}$ \\ 1. Departamento de Investigación en Física, Universidad de Sonora, Hermosillo, Sonora, México \\ 2. Doctorado en nanotecnología, Departamento de Física, Universidad de Sonora \\ 3. Departamento de Física, Universidad de Sonora, Hermosillo, Sonora, México
}

Extend the lubricant oil life is pursuit for scientific society, it represent a preservation of natural sources and contamination for oil wasted, furthermore, the reduction of costs to industrial application and time of machinery operation it would be extended as well. Lubricant oil analysis play an important role in the proper functioning of industrial machinery such turbines, compressors and any engine.

In this work, are present preliminary results of monitoring the degradation of engine oil by surface plasmon detector, it which will be correlated with FT-IR technique by norm ASTM-E-2412, this technique is the most used to interpret the oil quality [1,2]. FT-IR can detect the evolution of the functional groups that are present in the lubricating oil and through them can predict the condition of the oil, the most representative groups for this characterization correspond to oxidation, nitration and sulfation [3], Therefore these will be correlated with the results of the measurement of the surface plasmon extinction band of silver nanoparticles deposited on polystyrene cells by the laser irradiation technique and measured in a UV-VIS Ocean optics USB 2000+.

The FT-IR spectra of the lubricating fresh oil and the series of samples were carried out with Perkin Elmer Spectrum Two according [3] and are illustrated in figure 1, in which the evolution of the functional groups can be observed by the following vibration bands:

a) $\mathrm{C}=\mathrm{O}$ stretching vibration centered at $1718 \mathrm{~cm}^{-1}$ namely Oxidation

b) $\mathrm{SO}_{\mathrm{x}}$ bending vibration centered at $1158 \mathrm{~cm}^{-1}$ namely Sulfatation for sulfate by-products.

c) $\mathrm{NO}_{\mathrm{x}}$ stretching vibration centered at $1600 \mathrm{~cm}^{-1}$ namely Nitration

In the spectrum other peaks are observed that are not object of study in this work, however, they correspond to the compounds that form the lubricant base, water, gasoline, etc.

Figure 2 shows the evolution of the oxidation vs driving distance, in which a linear trend can be observed, with these results of FT-IR it is sought to correlate the results obtained by the above-described plasmon medium.

The experimental development of the silver nanoparticles deposited in the polystyrene cell was by the following formulation: silver nitrate $15 \mathrm{mM}$, sodium citrate $9 \mathrm{mM}$ and deionized water, the reagents were mixed in the same order inside the polystyrene cell and were irradiated with a Ar ion laser of the MellesGriot brand, whose wavelength is $488 \mathrm{~nm}$ at a power of $114 \mathrm{~mW}$ [4]. The laser exposure time was 40 min and an area covered with silver nanoparticles was observed on both surfaces of the polystyrene cell, after that, the sample obtained was taken to an electronic scanning microscopy equipment (FE-SEM JEOL JSM-7800F) to observe its composition and morphology. Figure 3 confirms the presence of silver nanoparticles by EDS, the other peaks correspond to the chemical composition of the polystyrene cell, therefore, it show the morphology of the distribution of the nanoparticles on the polystyrene cell, which is uniform and shows nanoparticles whose size is in the order of 50-90 nm. Once confirmed the deposition of silver nanoparticles in the polystyrene cell is still working in the step of measuring the 
surface plasmon for each of the oil samples. The preliminary result is shown in Figure 4, where a shrinkage of the surface plasmon extinction band is obtained, which goes from $473 \mathrm{~nm}$ to $530 \mathrm{~nm}$ for the zero sample and the sample 1 respectively, therefore Is considered a promising result for the purposes of the present study $[5,6]$

\section{References}

[1] A. Agoston et al, Conference IEEE (2004) p. 463.

[2] L Guan et al, Sensors and Actuators A 168 (2011), p. 22

[3] ASTM E-2412-0, Standard Practice for Condition Monitoring of Used Lubricants by Trend Analysis Using Fourier Transform Infrared (FT-IR) Spectrometry. Standard Volume.03.06 (2005).

[4] Masanori Sakamoto et al. Journal of Photochemistry and Photobiology C. 10 (2009) 33

[5] M. Milanese et al, Sensors and Actuators A 223 (2015), p. 97

[6] Ricciardi A. et al. Sensors. Lecture Notes in Electrical Engineering, Volume 319. Springer, Cham (2015)

[7] The authors acknowledge the financing of CONACyT for project 255791-NFR 2015

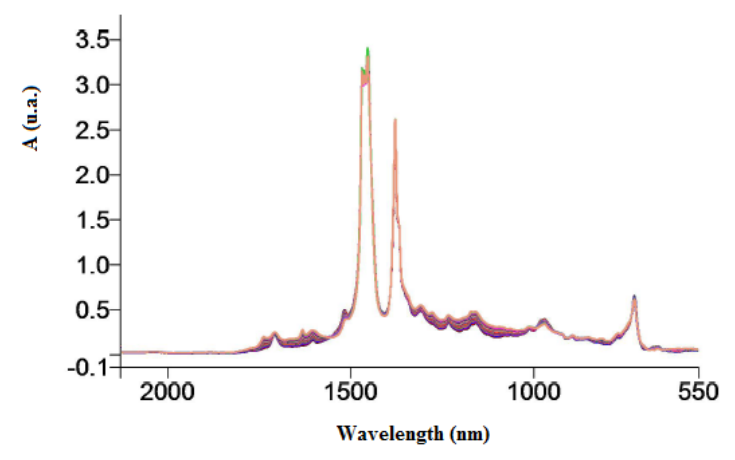

Figure 1. FT-IR spectra of fresh oil and the series samples.

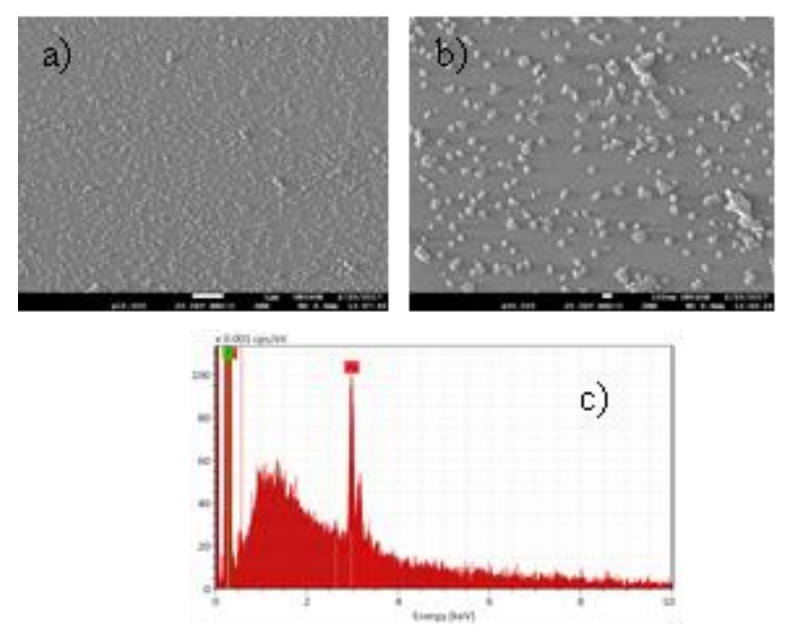

Figure 3. SEM micrographs a) and b) Morphology. c) Chemical composition by EDS

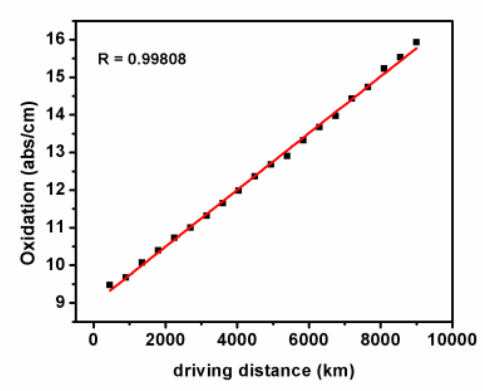

Figure 2. FT-IR evolution of the oxidation vs driving distance.

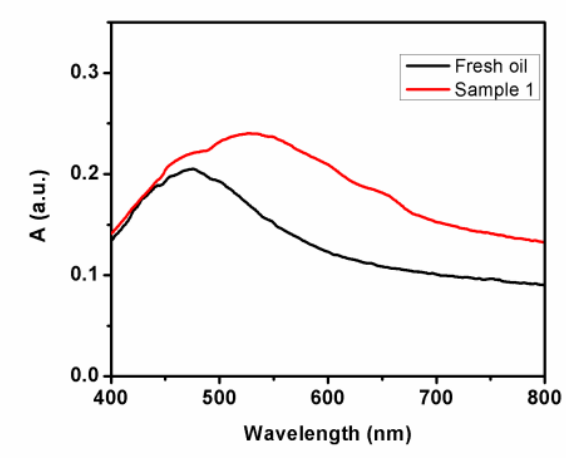

Figure 4. UV VIS - Surface plasmon extinction band for sample zero and one 